\title{
Nouvelles tendances de la muséologie
}

\section{François Mairesse}

\section{CpenEdition}

\section{Journals}

Édition électronique

URL : http://journals.openedition.org/iss/374

DOI : $10.4000 /$ iss. 374

ISSN : 2306-4161

\section{Éditeur}

ICOM - International Council of Museums

\section{Édition imprimée}

Date de publication : 1 décembre 2015

Pagination : 11-14

ISSN : 2309-1290

\section{Référence électronique}

François Mairesse, « Nouvelles tendances de la muséologie », ICOFOM Study Series [En ligne], 43b |

2015, mis en ligne le 06 février 2018, consulté le 21 décembre 2020. URL : http://

journals.openedition.org/iss/374 ; DOI : https://doi.org/10.4000/iss.374 


\title{
Nouvelles tendances de la muséologie
}

\author{
François Mairesse
}

Président d'ICOFOM - Université Sorbonne nouvelle - Paris 3, CERLIS, ICCA, France

Le thème choisi pour le $37^{\mathrm{e}}$ symposium international d'ICOFOM, organisé à Paris du 5 au 9 juin 2014, avait été envisagé de manière à ouvrir de nouvelles pistes de réflexion sur le futur de la muséologie. Evoquer les nouvelles tendances de la muséologie, c'est prendre acte du fait que le monde des musées, durant ces dernières décennies, a connu des changements considérables, à commencer par leur multiplication à travers le monde, mais aussi déjà leur transformation radicale, soit en matière de communication (le rapport à l'exposition ou le développement du musée comme média), soit en matière de transformation du patrimoine, de rapport au contemporain ou d'approche des publics. Le contexte économique (le développement de la logique néolibérale, mais aussi la crise économique), de même que les transformations technologiques (le développement d'Internet et du numérique) ont tout autant radicalement transformé la manière de penser le champ muséal. II ne paraît guère audacieux de supposer que ces transformations vont se poursuivre et entraîner des changements dans la manière de percevoir le champ muséal au XXI ${ }^{\mathrm{e}}$ siècle. C'est dans un tel contexte que l'appel à propositions, lancé à la fin de l'année 2013, fut envisagé de manière résolument ouverte, afin de recueillir des contributions suffisamment différentes pour tenter d'envisager, de manière globale, l'esquisse d'un panorama sur les sujets émergents dans le domaine de muséologie. Neuf thématiques furent ainsi proposées afin de suggérer un certain nombre de pistes de réflexion aux auteurs: la géopolitique de la muséologie ou les manières de penser le champ muséal à travers le monde, la muséologie en tant que discipline et matière d'enseignement, le rapport au patrimoine et la question des collections, l'éducation et la communication, la relation au contemporain, les contours de la cybermuséologie, la muséologie participative, l'éthique du champ muséal au $\mathrm{XXI}^{\mathrm{e}}$ siècle, enfin l'histoire et la prospective en matière de muséologie.

Le nombre de propositions reçues (plus de 200 abstracts) a nécessité une première sélection ; plus de 75 communications furent ainsi présentées au colloque de Paris. C'est encore une double sélection ultérieure qui a permis de rassembler les contributions éditées dans les volumes 43a et 43b d'ICOFOM Study Series.

Le foisonnement des sujets évoqués dans les différents articles a rendu assez difficile la répartition des sujets entre les deux volumes. Certaines des pistes suggérées furent nettement plus prisées que d'autres, imposant une réorganisation des thématiques. Certaines tendances se dégagent néanmoins de cet ensemble, qui nous ont permis de proposer la présente répartition en deux volumes, tant pour les articles théoriques que pour les études de cas (seconde partie de chaque volume). Pour des raisons de lisibilité, nous avons cependant préféré conserver l'ordre alphabétique à l'intérieur des volumes et des parties.

L'ICOFOM, depuis sa création, travaille essentiellement sur les aspects théoriques du champ muséal, à partir d'une approche 
globale intégrant l'ensemble des musées et des institutions qui lui sont proches, ainsi que l'ensemble des fonctions muséales (préservation, recherche, communication). Cette approche, qui trouve écho chez beaucoup de membres d'ICOFOM, n'est pas suivie par tous les chercheurs, tant s'en faut. Nombre de contributions présentées lors du colloque et reprises ici insistent plus précisément sur certains aspects spécifiques du musée: l'institution comme média, les expositions, la préservation, le rapport aux visiteurs, etc. Ce parti-pris nous a permis de regrouper, dans un premier volume (vol. 43a) les contributions visant essentiellement une approche générale de l'institution ou de la muséologie et de son évolution, tandis que nous avons tenté de regrouper, dans le second volume (vol. 43b) les contributions plus directement consacrées à l'une des approches du phénomène muséal, qu'il s'agisse du visiteur, des fonctions muséales ou des aspects éthiques qui leur sont liées. Nous nous rendons bien compte du caractère parfois factice de tels regroupements, de même qu'il nous a parfois été difficile de distinguer les articles théoriques et les études de cas à caractère plus spécifique ou plus pratique: la frontière entre théorie et pratique, on le sait, est loin d'être clairement établie, l'un se nourrissant continuellement de l'autre.

Le premier volume (vol. 43a) consacré aux communications du colloque reprend donc un certain nombre de questionnements généraux sur le devenir du champ muséal et de la muséologie. D'emblée, plusieurs contributions analysent les fondements ou l'histoire de la discipline afin de mieux comprendre son évolution possible. Afin d'explorer la muséologie au $\mathrm{XXI}^{\mathrm{e}}$ siècle, Deloche revient sur ses fondamentaux; Guzin s'interroge également sur l'un des thèmes chers à l'ICOFOM, la " muséologie de l'Est ", tandis que Brulon Soares évoque la Nouvelle muséologie; Menezes de Carvalho et Scheiner interrogent la muséologie à partir de la la notion bourdieusienne de champ; de manière plus pratique, Cardonna analyse les publications en muséologie, tandis que Gachet retrace l'histoire de la Lettre de l'OCIM. Sur le plan de la formation, Bergeron et Carter s'interrogent sur l'évolution de la muséologie et ses répercussions sur sa manière de l'enseigner. Julião analyse le concept d'historicité dans ses liens avec le musée et Nomiku s'interroge sur l'intégrité de la muséologie. La muséologie se conçoit et s'enseigne, on le sait, de manière différente dans le monde. Costa, dans cette perspective, évoque le concept de "muséologie du sud », tandis que de Melo, Menezes de Carvalho et de Moraes analysent l'idée d'une " muséologie amazonienne ». Brulon Soares, Menezes de Carvalho et de Vasconcelos interrogent enfin, de manière plus pratique, les différents courants de la muséologie au Brésil, tandis que Sustar analyse les musées pédagogiques.

Deux éléments particuliers ont eu une influence considérable sur la manière de penser le futur de la muséologie: l'évolution du numérique, d'une part, celle des mécanismes économiques de l'autre. On sait l'influence d'Internet et des technologies de l'information et de la communication sur le musée et la manière de penser son évolution. La cybermuséologie constitue l'une des possibilités d'envisager les nouveaux contours du champ muséal, ce qu'évoquent Langlois et, de manière plus pratique, Leshchenko. L'évolution de la manière de concevoir les mécanismes économiques constitue également une donnée de première importance pour comprendre notamment les relations entre le musée et le marché de l'art, ce que montre Doyen à travers le patrimoine ethnographique; mais c'est surtout la crise économique et, 
parallèlement, le développement de logiques collaboratives et participatives qui a le plus largement influencé la pensée muséale contemporaine : les contributions d'Agostino, de Moolhuisen et de Radice, entre autre, témoignent de l'importance actuelle de ce mode de fonctionnement particulier de l'institution.

Le second volume (vol. 43b) évoque plus directement la muséologie à partir des fonctions muséales ou du point de vue de sa réception, notamment à partir de l'angle des études de visiteurs. La question du fonctionnement du champ muséal et particulièrement celui du musée, induit la réflexion éthique, ce qui s'inscrit entre autres au cœur du propos de Maranda et d'Avila Mélendez. L'éthique suppose l'examen des fins et notamment la réflexion sur les publics ou les utilisateurs (potentiels, actuels et futurs) du musée. II n'est guère étonnant que la question des visiteurs et des publics constitue l'une des portes d'entrées les plus obligées en matière de recherches sur le champ muséal, notamment à travers les études de publics, en témoignent les interventions de Harris, de Mijalovic et Romanello, de Schmitt, de Romanallo, de Crenn et Roustan, ou encore de Jutant et Lesaffre. La relation entre le musée et son public suppose, en quelque sorte, le principe du musée comme système de communication, ce sur quoi reviennent, de manière plus pratique, Chuvilova et Shelengina d'une part, Roda de l'autre. Ce lien particulier entre l'institution muséale et son public repose sur l'une des fonctions classiques du musée, résumée à travers le principe général de communication, mais plus généralement évoqué à travers les notions d'éducation ou, plus récemment, de médiation et d'inclusion. Cette perspective a été choisie par de nombreux auteurs, qu'il s'agisse de Cornélis et Jaminon, de Dufresne-Tassé et O'Neil, insistant sur les best practice en matière d'éducation et de médiation, mais aussi de Sant'Anna de Godoy (pour les groupes de jeunes et adultes et les programmes d'alphabétisation), de Garcia Ceballos (pour les groupes plus âgés), de Fontal et Marin (pour les programmes d'inclusion) ou de Thévenot et al. (pour les digital natives), tous témoignent des spécificités de l'institution en regard des groupes auxquels celle-ci s'adresse.

Après l'éducation ou la médiation, l'analyse des expositions constitue également une voie à partir duquel le champ muséal est régulièrement investigué, qu'il s'agisse de techniques ou d'expériences particulières liées à la mise en exposition, comme les étudient Schärer, Noël-Cadet et Bonniol ou De Caro et, de manière plus pratique, Chang et Shibata, ou de questionner la mise en exposition de l'exposition elle-même, comme le propose Camart. La question de la préservation (et plus spécifiquement celle de la conservation), sujet pour le moins important, fait également l'objet d'analyses spécifiques: la figure du conservateur est abordée par Hoffman, Jones et Burns, tandis que Smeds et Angilis questionnent d'une part les questions de préservation à l'aune de la question des déchets, d'autre part la préservation des patrimoines immatériels, à travers les récits de vie. La question de l'aliénation, enfin, est évoquée à travers une étude des musées finlandais par Robbins.

Comme on peut le constater, un grand nombre des thèmes présentés semblent s'inscrire à la suite de réflexions initiées depuis parfois plusieurs années. Est-il, à ce stade, possible de parler de nouvelles tendances se dégageant de cet ensemble ? II est évident que ces dernières n'émergeront dans la plupart des cas que de thématiques déjà connues, même si le goût pour la nouveauté radicale nous pousse au renouvellement des concepts, pour le 
meilleur et, parfois aussi, pour le pire. Par ailleurs, la diversité d'origine des contributions, écrites dans l'une des trois langues de travail de l'ICOM, témoigne des différences importantes existant au sein du monde de la muséologie, tant pour ce qui concerne leur stade de développement que pour leur rapport au patrimoine ou aux publics, mais aussi pour ce qui concerne les fondements même de la muséologie. C'est sans doute l'étude de cette diversité qui constitue l'essence du travail d'ICOFOM: rassembler, cartographier et synthétiser l'ensemble des propos liés au champ muséal. En ce sens, la publication de ces actes ne fait qu'initier le travail d'étude des tendances qui se dégagent de la muséologie actuelle, travail continu et forcément sans fin, mais qui participe pleinement d'une certaine idée de la recherche scientifique et du travail du chercheur. 